\title{
A new timing detector for the CT-PPS project
}

\author{
R. Arcidiacono ${ }^{\mathrm{a}, \mathrm{b}, *}$, on behalf of the CMS and TOTEM collaborations \\ ${ }^{a}$ INFN - Torino, Italy \\ ${ }^{b}$ Università del Piemonte Orientale, Italy
}

\begin{abstract}
The CT-PPS detector will be installed close to the beam line on both sides of CMS, 200 meters downstream the interaction point. This detector will measure forward scattered protons, allowing detailed studies of diffractive hadron physics and Central Exclusive Production. The main components of the CT-PPS detector are a silicon tracking system and a timing system. In this contribution we present the proposal of an innovative solution for the timing system, based on Ultra-Fast Silicon Detectors (UFSD). UFSD are a novel concept of silicon detectors potentially able to obtain the necessary time resolution $(\sim 20 \mathrm{ps}$ on the proton arrival time). The use of UFSD has also other attractive features as its material budget is small and the pixel geometries can be tailored to the precise physics distribution of protons. UFSD prototypes for CT-PPS have been designed by CNM (Barcelona) and FBK (Trento): we will present the status of the sensor productions and of the low-noise front-end electronics currently under development and test.
\end{abstract}

\section{Introduction}

The main physics goal of the CT-PPS project (CMS-TOTEM Precision Proton Spectrometer) is the study of Central Exclusive Production $p p \rightarrow p X p$ (CEP) at the LHC. The distinctive signature of CEP events is to have, concur5 rently to the hard process seen in the CMS detector, two protons in the final state, scattered at very small angles with respect to the beam lines. Figure 1

${ }^{*}$ Corresponding author

Preprint submitted to Nuclear Instruments and Methods A

May 27, 2016

(C) 2016. This manuscript version is made available under the Elsevier user license http://www.elsevier.com/open-access/userlicense/1.0/ 
shows, as an example, the Feyman diagram for the CEP of di-lepton events. To distinguish this category of events, one needs to detect the outgoing protons, measuring with high precision their momentum, direction and timing. The energy and scattering angle are directly related to the kinematic variables $\xi$, the fractional longitudinal momentum loss of the outgoing proton, that identify the mass of the central event.

In order to achieve this, the CT-PPS project foresees to install near-beam detectors in the very forward regions downstream of CMS, on both sides of the Interaction Point (IP5). In particular there will be:

- a proton spectrometer made of two tracking pixel stations, making use of the beam line magnets;

- one station with timing detectors.

All proposed detectors will be housed inside horizontal Roman Pots placed 20 in the 200-220 m region, along the beam line. The Roman Pots are provided by the TOTEM collaboration, which has acquired an excellent experience in the past three years. The CT-PPS project plans to be completed and start collecting physics data in normal CMS runs in 2017 (possibly already during 2016).

In the following sections, we will describe the requirements of the timing system and present in detail the new proposal based on a novel type of silicon sensors (UFSD).

\section{Requirements of the timing system}

The measurement of the arrival time of the protons will be an important handle to be able to associate them to the correct interaction vertex, reducing the background from pileup at high luminosity. In fact, in the next two years, the average number of collisions per bunch crossing will be up to 50, spread out along the $z$ direction within few centimetres. A precision of 20 ps on the time of each proton will determine the vertex position with a precision of $\sim 4.2 \mathrm{~mm}$, 


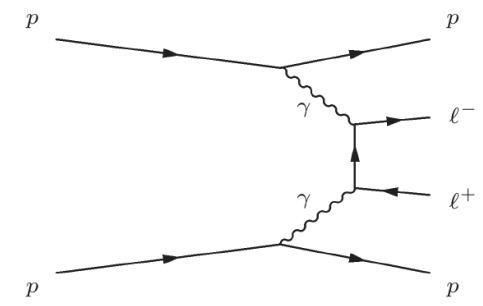

Figure 1: Feynman diagram for a central exclusive production of a pair of leptons in a p-p collision

using the $z$-by-timing method $\left(z=c\left(t_{1}-t_{2}\right) / 2\right)$. This resolution on the vertex is enough to keep the majority of the events.

For what concerns the operational requirements, the detectors of the timing system have to be inserted in the secondary vacuum inside the TOTEM Roman Pots, and be brought as close as possible to the beam line. They need to be 40 small area detectors $\left(\leq 2 \mathrm{~cm}^{2}\right)$ :

- with reduced dead region: with slim edge or edgeless towards the beam;

- segmented accordingly to the hit density distribution, to reduce to the minimum the probability of double hits in the same channel;

- radiation hard: the expected fluence in the area closest to the beam is $\sim 2 \times 10^{13} \mathrm{neq} / \mathrm{cm}^{2}$ per $\mathrm{fb}^{-1}$.

\section{CT-PPS timing detector: new proposal based on UFSD.}

The baseline for the timing detector is based on diamonds, adapted from the ongoing TOTEM upgrade program. The installation of diamond planes mounted in the cylindrical RP (horizontal) is foreseen for early summer 2016.

50 In parallel there are other solutions pursued, based on fast silicon detectors and on quartz bars $\mathrm{R} \& \mathrm{D}$ programs.

Here we focus on the ongoing work in the context of the UFSD R\&D program, where the UFSD state-of-the-art prototype (measuring only the time) is 
tailored for the CT-PPS case. This would be the first demonstrator of UFSD.

The proposal for this detector is to have planes of thin segmented Low Gain Avalanche Diode (LGAD) sensors, the core technology of UFSD, with adapted strip size. LGAD sensors [1, 2, 3] are sensors with a thin (tens of microns) layer of moderate gain, right below the electrode, at the $n-p$ junction. The gain is obtained by implanting an appropriate charge density that locally generates very high fields $\left(N_{D} \sim 10^{16} / \mathrm{cm}^{3}\right)$. Figure 2 shows the internal structure of an LGAD sensor with the typical electric field profile: charge multiplication occurs in the gain layer, providing a large $d V / d t$ signal, well suitable to measure time accurately.

Current simulations, based on the WF2 package 4, of the properties of signal formation in LGADs are well confirmed at test-beams for 300-micron thick sensors. The simulation also forecasts an increasing improvement with thinner sensors and higher gain, reaching $\sigma_{t} \sim 30 \mathrm{ps}$ for a thickness of 50 microns and gain around 15-20, as shown in Figure 3 .

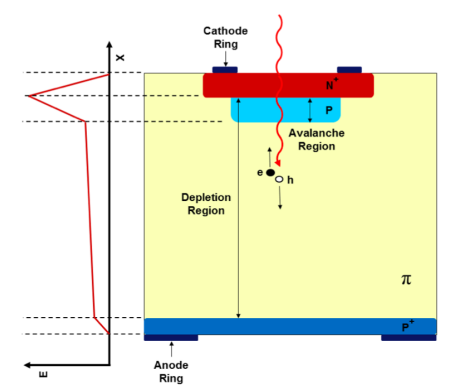

Figure 2: Schematic view of a Low-Gain Avalanche Diode with electric field profile.

For what concerns the radiation hardness of the current UFSD sensors, stud70 ies show that highly irradiated LGADs (so far 300-micron pads) have a progressive decrease of signal amplitude for fluences above $10^{14} \mathrm{neq} / \mathrm{cm}^{2}$, with a complete disappearance of the gain at $10^{15} \mathrm{neq} / \mathrm{cm}^{2}$, as can be seen in Figure $4[5]$. This effect has not been fully understood yet. Two possible hypotheses are under study: (i) an inactivation of acceptors (Boron) of the gain layer due to 


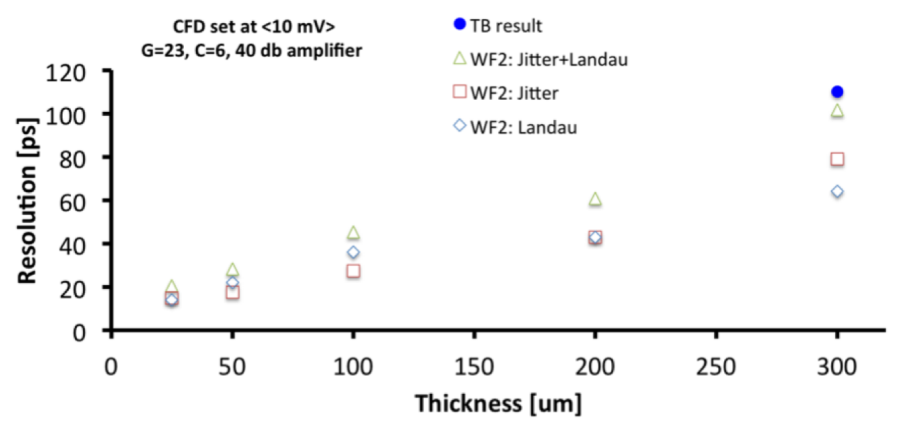

Figure 3: Total time resolution (with contributions from jitter and Landau fluctuations) of an LGAD with gain=23, as a function of the sensor thickness: WF2 predictions and test beam results.

radiation defects, and (ii) a dynamic reduction of the gain layer doping due to charge trapping (induced by high leakage currents).

There are attempts to produce LGADs with more radiation hard doping elements for the gain layer (Gallium). For the second case, thinner detectors operated at low temperature have a much reduced leakage current and should be intrinsically less sensitive to the dynamic charge injection effect.

\subsection{The characteristics of the sensor}

Considering the detector requirements and the match with the UFSD prototypes currently under development, the proposal for the CT-PPS timing detector foresees a thin LGAD sensor (50-micron thickness, gain $\sim 15)$, with an area

of $12 \times 6 \mathrm{~mm}^{2}$, slim edge of 200 microns on the side facing the beam, segmented in $16 \times 2$ pixels as detailed in Figure 5 . The 16 pixels closer to the beam are half size (and half capacitance) with respect to the other 16 .

One timing station will be composed of a stack of 4 to 6 planes. The layout of the 32 channels is asymmetric, such as to guarantee no inactive gap aligned, when composing the stack with half of the planes rotated by 180 degrees in the vertical direction.

The expected time resolution for one such plane is of $30 \mathrm{ps}$. 


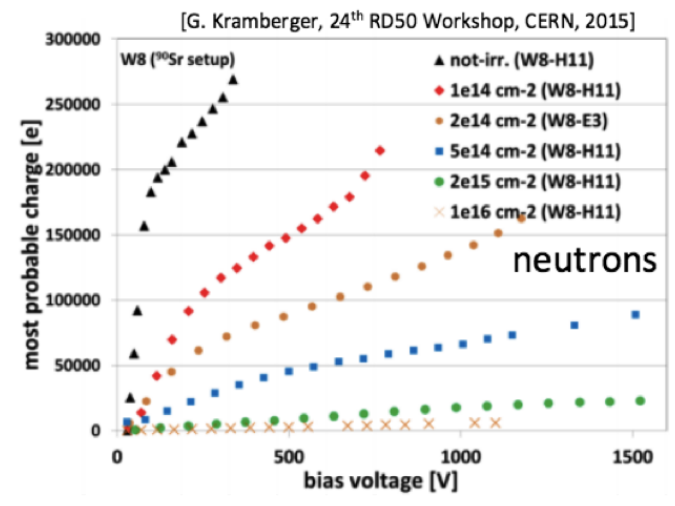

Figure 4: Charge collection as a function of the bias voltage for LGAD pads (300-micron thick) irradiated with neutrons at different fluences

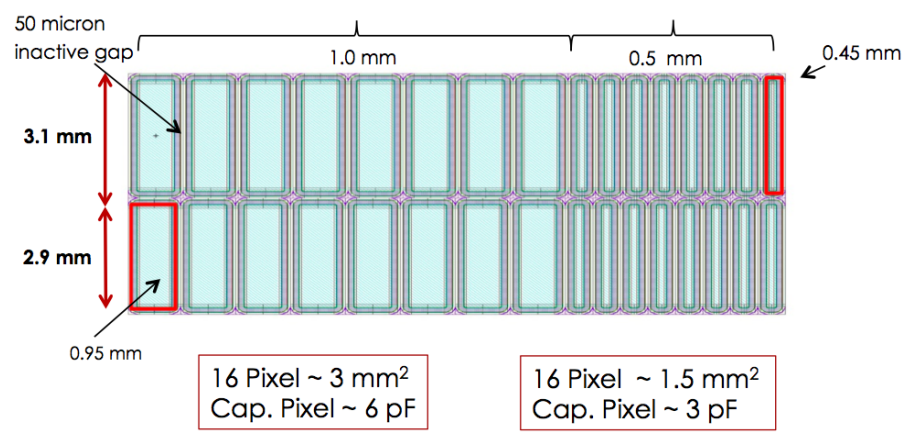

Figure 5: UFSD sensor for the CT-PPS timing detector: CNM mask

\subsection{Status of CT-PPS sensor production}

Several new LGAD sensor productions geared towards the CT-PPS project are ongoing at different stages of processing and will be available in the next 6 months.

In the framework of the RD50 collaboration, CNM (Centre Nacional de Microelectronica, Barcelona) is producing various batches of LGAD wafers, with multiple geometries (pads/pixels/strips) and characterized by various thicknesses. Two productions (FZ and EPI substrates), 50 micron thick, have the CT-PPS sensor with the final layout. The first production (FZ) is expected to 
be completed in May.

In the framework of the ERC-UFSD grant and INFN group V, FBK (Fondazione Bruno Kessler, Trento) is also working on the design of LGAD sensors:

the first production, 300-micron thick, is expected in spring 2016. A second batch, 50-micron, housing the CT-PPS design is in preparation and is expected to arrive in September (including some wafers with Gallium doping for radiationhardness studies).

\subsection{Status of the signal processing chain}

For what concerns the signal processing chain of the proposed detector, given the short time scale of the project, the idea is to be compatible as much as possible with the read-out system prepared for the diamond detectors (baseline), including the mechanical structure hosting the devices inside the RP.

The existing chain is composed of: a preamplifier; a discriminator circuit (NINO board) [6] measuring the Time-Over-Threshold (TOT) according to a user-defined threshold; a digitizer board with a High Precision TDC (HPTDC); interface to the DAQ. All components from the discriminator onwards, housed outside the RP, can be used once the stage of pre-amplification is adapted to the UFSD signal. The expected time resolution of the full chain, detectorpreamplifier-discriminator-TDC is of the order of 40-50 ps per plane.

There are currently two alternatives pursued in parallel for the first stage of the read-out system:

- preAmp + NINO: a discrete components solution where the detector output is fed to a surface mounted preamplifier that matches NINO inputs. A first prototype board of a low noise, distortion-free BroadBand preamplifier, based on the PSA4-5043 monolithic, has been already tested in the lab with very promising results, as can be seen in Figure 6. The final board with 32 channels where the sensor will be wire-bonded will arrive in spring 2016.

- ACC (Analog Comparator Chip): this option goes towards a more suitable 
implementation of the front-end part, with the realization of a custom analog ASIC for the amplification and discrimination stage. The chip is currently under development and aims to replace the discrete amplifier and the NINO stage, matching directly the HPTDC inputs. TCAD simulations show an excellent noise performance, with a noise of 500 ENC for a 6 $\mathrm{pF}$ detector (slope $50 \mathrm{ENC} / \mathrm{pF}$ ). The design of the comparator has been completed, with the implementation of Time Over Threshold for the time walk compensation. This chip will be submitted in May and ready to be tested in July.
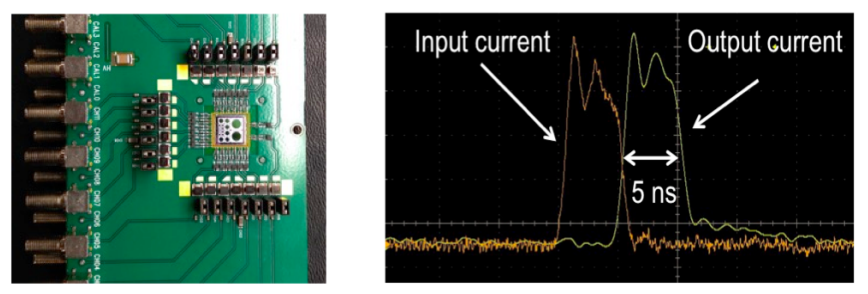

Figure 6: Picture of the prototype board with BroadBand amplifier reading a UFSD pad matrix (left). On the right: comparison between the sensor signal and the output of the amplifier (attenuated), measured with a high-precision oscilloscope.

\section{Conclusions and outlook}

An alternative solution for the timing system of CT-PPS has been presented. It is based on the recently conceived UFSD detectors, LGAD sensors optimized for time measurements, in rapid development phase. They look very promising in terms of ultimate time resolution: $\sim 30$ ps for 50-micron thick sensors with a gain of 15-20. The radiation hardness of such devices is still under study. The current design is working up to $10 \mathrm{fb}^{-1}$ (300-micron sensors at room temperature). On both sensors and front-end electronics there are many developments ongoing in parallel: the next 6 months will determine the success of this first application of Ultra Fast Silicon Detectors. 


\section{Acknowledgements}

This project has received funding from the European Research Council (ERC) under the European Unions Horizon 2020 research and innovation programme (669529) and from the U.S. Department of Energy (grant number DESC0010107).

[1] P. Fernandez-Martinez, et al., Low Gain Avalanche Detectors for high energy physics, in: Proceedings, 10th Spanish Conference on Electron Devices, Vol. 2015, 2015. doi:10.1109/CDE.2015.7087475.

[2] G.-F. D. Betta, et al., Design and tcad simulation of double-sided pixelated low gain avalanche detectors, NIM A 796 (2015) $154-157$.

[3] N. Cartiglia, et al., Design optimization of ultra-fast silicon detectors, Nucl. Instrum. Meth. A796 (2015) 141-148. doi:10.1016/j.nima.2015.04.025

[4] F. Cenna, et al., Weightfield2: A fast simulator for silicon and diamond solid state detector, NIM A 796 (2015) $149-153$.

[5] G. Kramberger, et al., Radiation effects in Low Gain Avalanche Detectors a after hadron irradiations, JINST 10 (07) (2015) P07006. doi:10.1088/ 1748-0221/10/07/P07006.

[6] F. Anghinolfi, et al., NINO: An ultra-fast and low-power front-end amplifier/discriminator ASIC designed for the multigap resistive plate chamber,

170 „ Nucl. Instrum. Meth. A533 (2004) 183-187. doi:10.1016/j.nima. 2004. 07.024 